\title{
Molecular Mechanism of Intercalation of SOX-4 Protein into DNA Inducing Bends and Kinks
}

\author{
Reman Kumar Singh and Arnab Mukherjee*
}

Department of Chemistry, Indian Institute of Science Education and Research, Pune-411008, India.

\section{Unrestrained simulation on complexes A-1, A-2 and A-3}
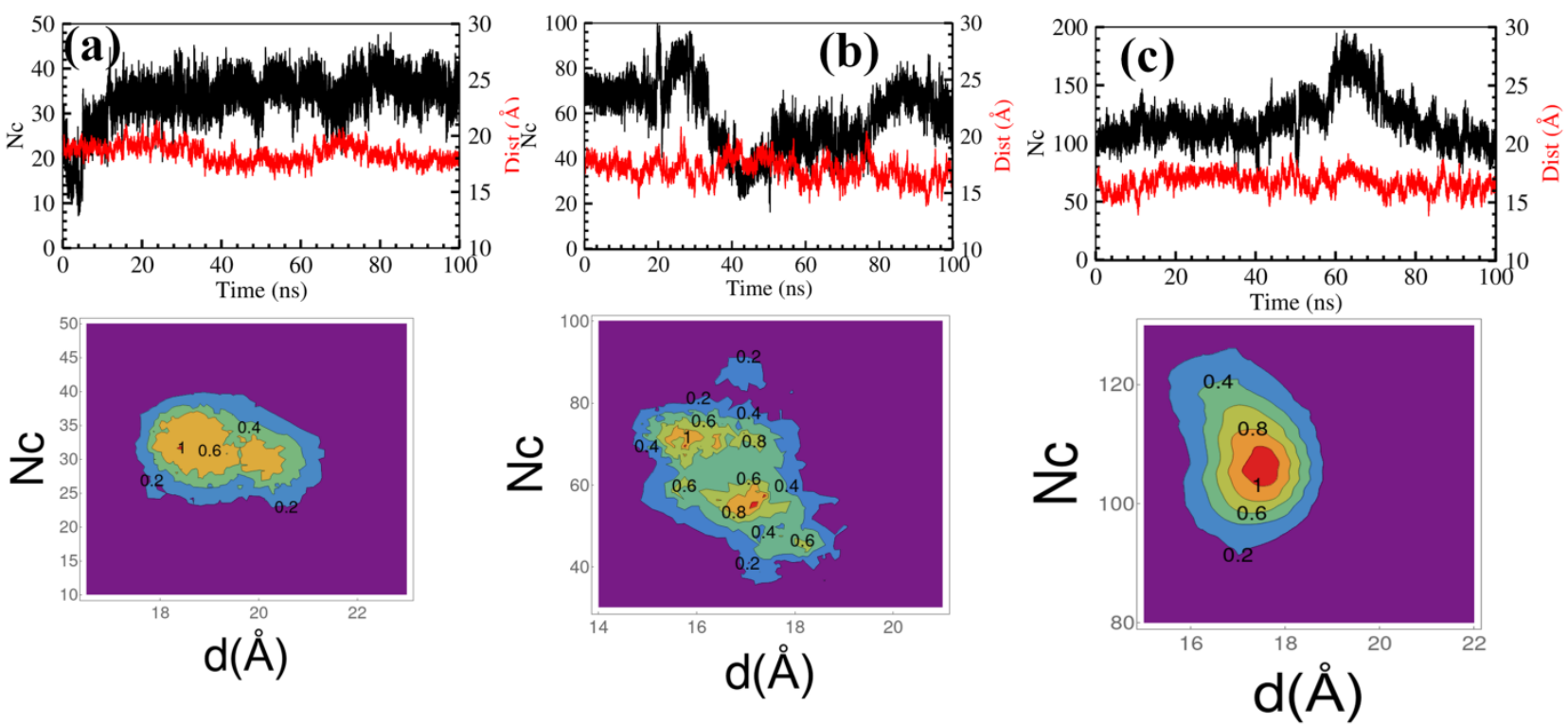

Figure S1. Upper panel shows the time variation of the native contact (black, left axis) and distance (red, right axis) between SOX4-DNA for the conformation (a) A-1 (b) A-2, and (c) A-3. Bottom panel shows the corresponding 2D distributions of $\mathrm{Nc}$ and distance. The mean and standard deviation of Nc and distance are $29 \pm 9.6$ and $18.6 \pm 0.9 \AA$ for $A-1,59 \pm 20$ and $17.0 \pm 1.2 \AA$ for $\mathrm{A}-2$ and $107 \pm 22$ and $17.6 \pm 1.3 \AA$ for $\mathrm{A}-3$.

\section{Mechanism: binding and rotation of Protein}

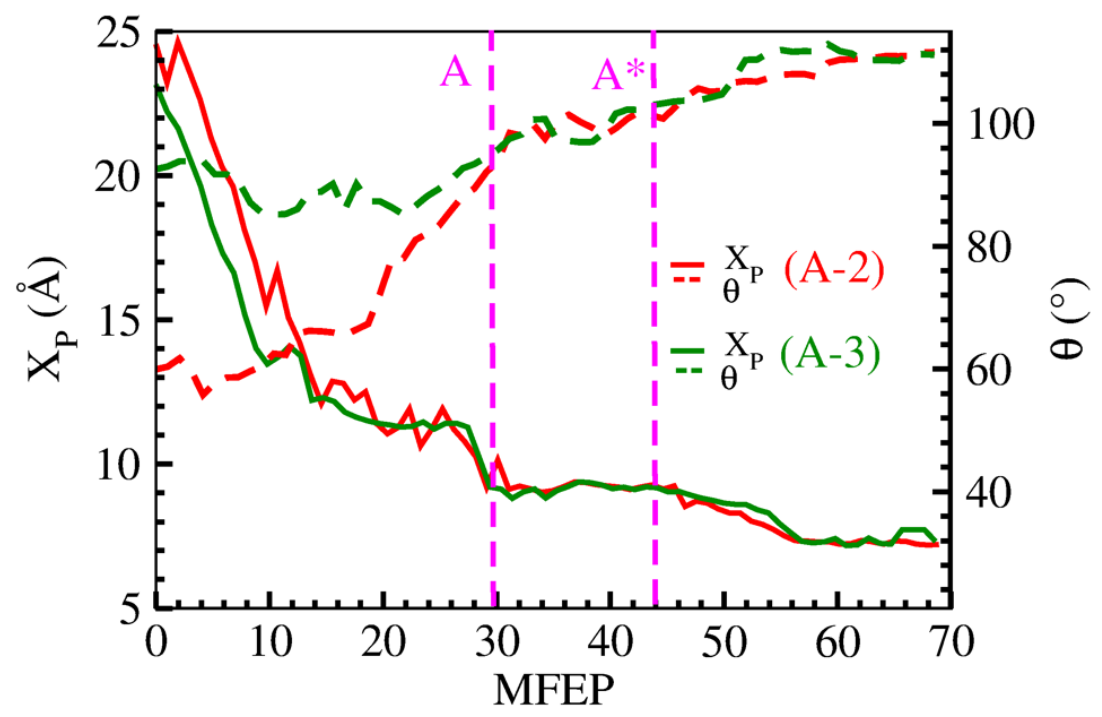

Figure S2. Variation of $\mathrm{X}_{\mathrm{P}}$ (thick lines, left axis) and rotation angle $\theta$ (dashed lines, right axis) along protein binding pathways for less probable complexes, A-2, and A-3. See the manuscript 
for the definition of $\mathrm{X}_{\mathrm{P}}$ and $\theta$. Vertical dashed line show the location of nonspecific ("A") and specific (" ${ }^{*}$ ") associated states along the MFEP.

\section{Contact residue maps for different conformations at different stages of binding.}

We have calculated the SOX4-DNA contact residue pairs (defined to be a distance less than $4.5 \AA$ from each other) at the $\mathrm{A}, \mathrm{A}^{*}$, and I states (denoted by red circles) and compared them with the same for the crystal structure (blue triangles) in Fig S3. The more overlap between the red circles and blue triangles indicates similarity in the geometry of the configuration with the crystal geometry. For the complexes A-1, at A state, there are very less overlap as only ARG $(5,19)$ and LYS 4, 20,76) are in close contact. However, for A-2 and A-3, most of the pairs are overlapping with the crystal geometry. Also Fig 7e of manuscript shows the number of contacts between DNA and protein's charged and neutral amino acids. Fig 7e also shows that for complex A-1, only charged amino acids come in contact with DNA at "A" stage, whereas, A-2 and A-3 have more contacts even at that stage (including charge and neutral residue). Interestingly, even though the pair residues between SOX4-DNA is very different for A-1, A-2, and A-3 at all binding stages, they all converge to the same pair residues at the intercalated state ("I") (Fig S3(l, o, r)).

MFEP
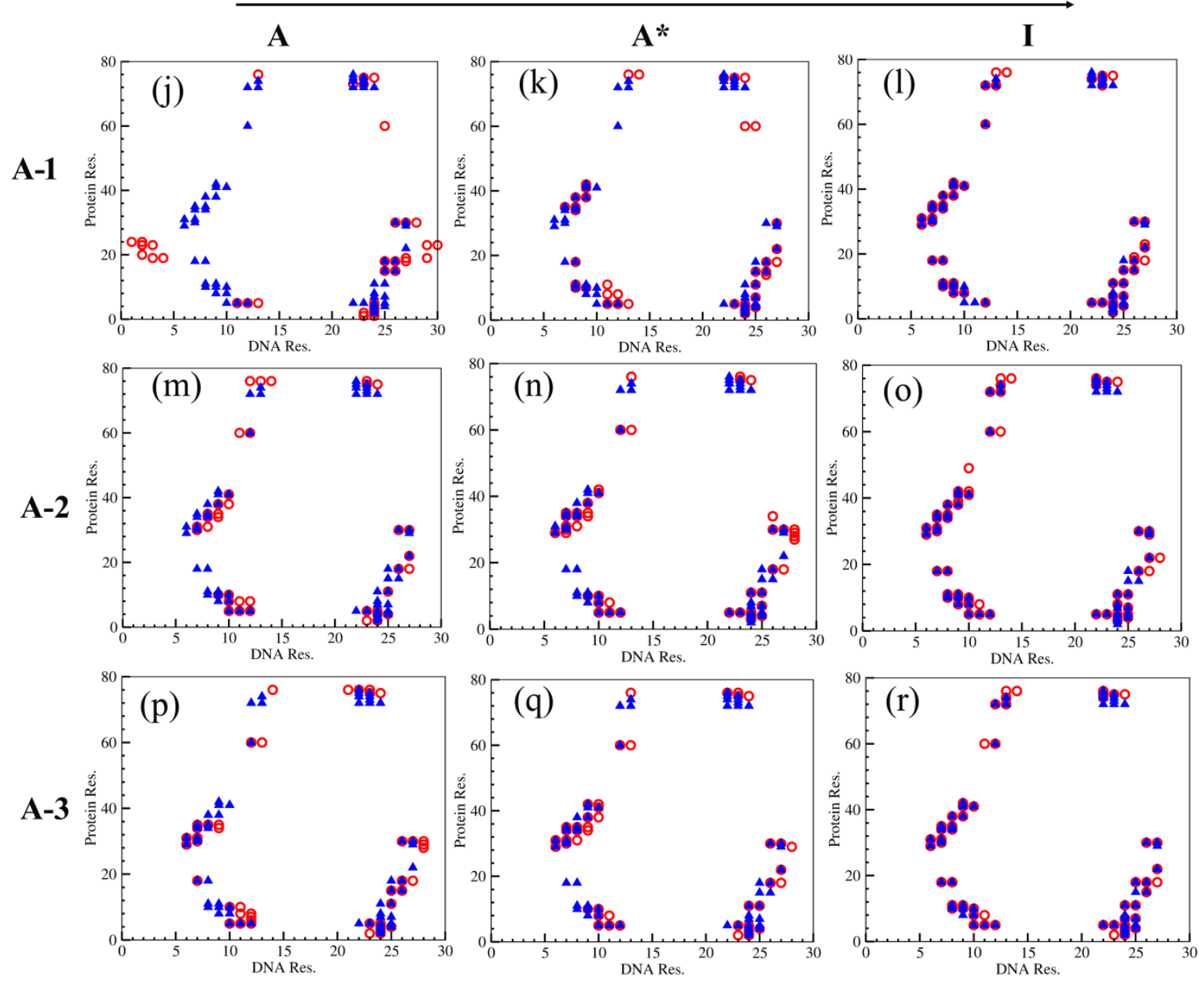

Figure S3. Contact residues (red circles) of SOX4 and DNA (within $4.5 \AA$ ) for three conformers A$1, A-2$, and $A-3$ at different binding stages (A, $\left.A^{*}, I\right)$ and crystal geometries (blue triangles). MFEP represent the minimum free energy path on which we have calculated the pair residues. Note that, at the "I" or intercalated stage of binding obtained from simulation, the contact residue pairs are almost overlapping on the contact residue pairs in the crystal geometry. 


\section{Structural change during the association of protein}

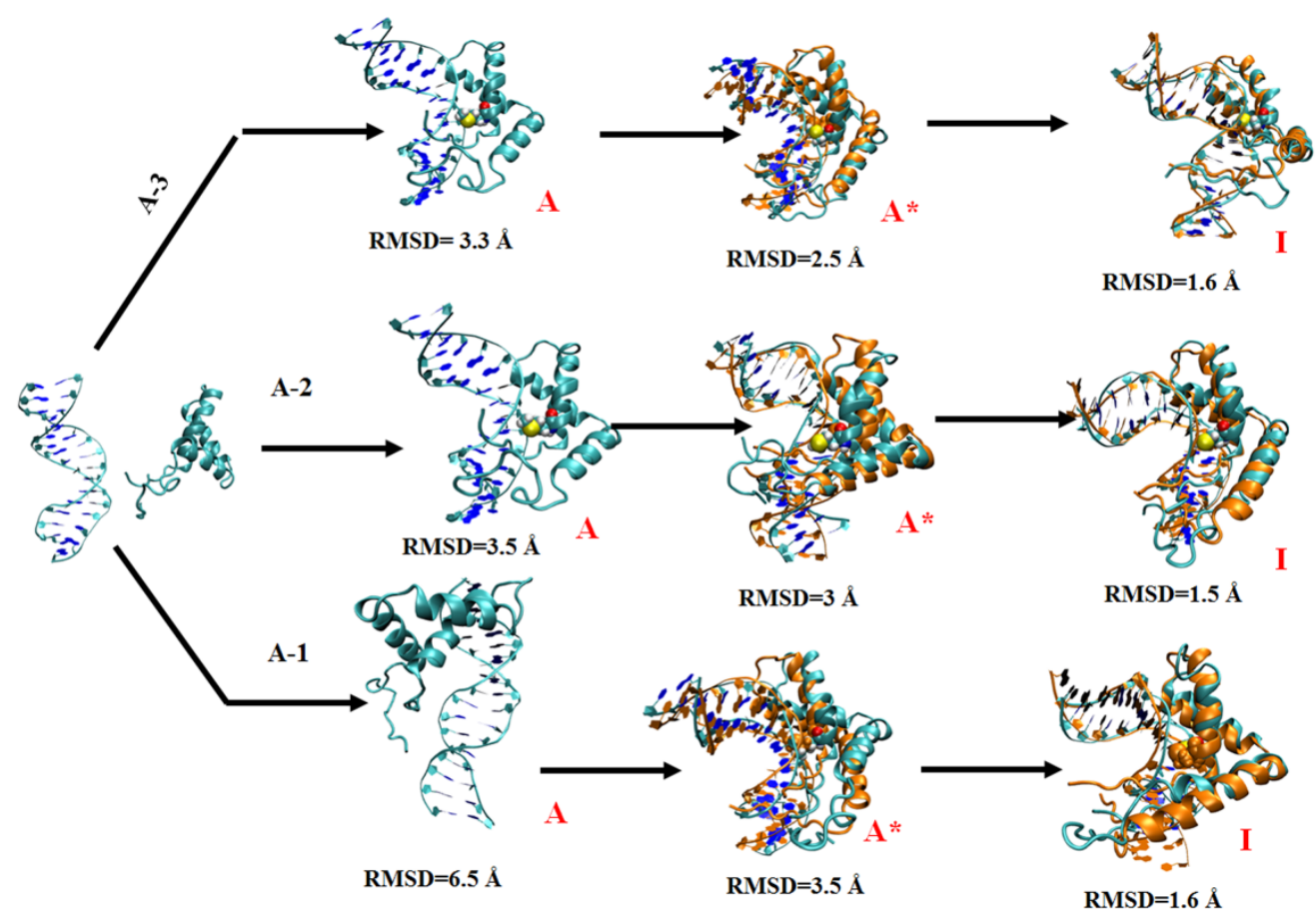

Figure S4. Structural changes in the DNA-protein complexes along protein association pathways. The protein's structure from simulation is shown in light green, while the orange color represents the crystal geometry. The intercalating amino acid MET39 is shown using van der Waals (vdw) representation. 